\title{
Rechtsgeschichte
}

http://www.rg-rechtsgeschichte.de/rg7

$\operatorname{Rg} 2005 \quad 33-37$

Zitiervorschlag: Rechtsgeschichte Rg 7 (2005)

http://dx.doi.org/10.12946/rg07/033-037

\section{Mahidé Aslan}

\section{Rückfahrkarte}

Das schweizerische Zivilgesetzbuch in der Türkei 


\section{Abstract}

The young republic of Turkey of 1922 was obliged By an international convention to establish a modern legal system. Very quickly the Swiss Civil Code was translated and enacted in Turkey. This article traces the fate of Swiss marriage law within Turkish society and shows how the newly imported law was largely ignored and disregarded by the people. Even many special legislative acts in the following years were not successful at enforcing the new marriage rules. In 2001a revision of the Turkish(-Swiss) Civil Code took place which once again referred to contemporary Swiss law. Though the legal transfer from Switzerland to Turkey seems clearly to have failed, it has Had some impact on the evolution of Turkish law. 


\section{Rückfahrkarte}

Das schweizerische Zivilgesetzbuch in der Türkei

»[Es] besteht für einen Staat, der um den Eintritt in die zivilisierte Gesellschaft kämpft, der Zwang zur Aneignung der Erfordernisse dieser Gesellschaft. " ${ }^{\mathrm{I}}$

So äußerte sich der ehemalige Justizminister Mahmut Esat Bozkurt in der Festschrift zum I5. Geburtstag des türkischen Zivilgesetzbuches. Bozkurt war Mitglied der Kommission gewesen, die mit der Aufgabe betraut worden war, ein Zivilgesetzbuch zu (er-)finden, das für die neue Republik gelten sollte.

Das Gesetzbuch, das die Kommission vorbereitet hatte, war kein neu erschaffenes, keine Sammlung von Normen, die das zu regeln suchten, was in der neuen türkischen Gesellschaft zu regeln war. Der Entwurf, den die Kommission vorlegte, war eine Übersetzung des schweizerischen Zivilgesetzbuchs. Die französische Fassung dieses dreisprachigen Gesetzeswerkes wurde durch die Kommission, bestehend aus Abgeordneten, Lehrern, Richtern und Anwälten, übersetzt und mit einigen Änderungen zu einem neuen Gesetzbuch geformt.

Nachdem 1922 die Republik ausgerufen worden war, sollte I923 die völkerrechtliche Anerkennung durch den Vertrag von Lausanne folgen, welcher der neuen Türkei unter anderen Verpflichtungen die Reorganisation des Rechtsund Justizwesens auferlegte. Dieses Vorhaben stand einerseits zeitlich unter Druck, da die völkerrechtliche Auflage rasch erfüllt werden musste. Eine Einmischung von Außen sollte vermieden werden. Andererseits fehlten im Land die Mittel und Strukturen, die erforderlich gewesen wären, um die Reorganisation professionell durchzuführen.

I Abgedruckt in: ERnst E. Hirsch,

Rezeption als sozialer Prozess,

Berlin I98I, 32 .
Die bestehende Ordnung hatte ihren Ursprung in der alten, osmanischen, und gerade diese Vergangenheit sollte abgeschüttelt werden. Die Regierung entschied, ausländische Gesetze $\mathrm{zu}$ übernehmen, um in kurzer Zeit moderne Gesetze als Landesrecht vorweisen zu können. Man bediente sich unter anderem in Italien, Deutschland und - für das Zivilrecht - eben in der Schweiz. Die Übersetzung des ZGB trat mit einigen Veränderungen als »Türkisches Zivilgesetzbuch « am 4. April 1926 in Kraft und blieb bis zum 3 I. Dezember 200I in Geltung.

Das Rezept hört sich einfach an: Man nehme ein Gesetzbuch, übersetze es und lasse es in Kraft treten. Welche Erfolge und Misserfolge ein solcher Transfer zeitigen kann, soll im Rahmen eines Forschungsprojekts untersucht werden. Die folgenden ersten Überlegungen gelten dem politischen und gesellschaftlichen Kontext des Rechtstransfers sowie einem Beispiel aus dem Familienrecht.

\section{Die Gründung der Republik} und die Gesetzesübernahme

Die Jahre der Republikgründung I922/I923 gingen mit einer Bündelung von Entscheidzwängen einher; ein spezifischer "Entschärfer « der Situation oder »Entscheider « aber fehlte: Vom großen Reich der Osmanen war ein Rumpf übrig geblieben, und selbst dieser war in seinem territorialen Bestand bedroht. Die Kriege hatten die Bevölkerung verkleinert, und der militärische Nachwuchs war geschrumpft. Die Bevölkerung sollte und musste wachsen. Die völkerrechtliche Anerkennung sollte sichern, was vom Osmanen-

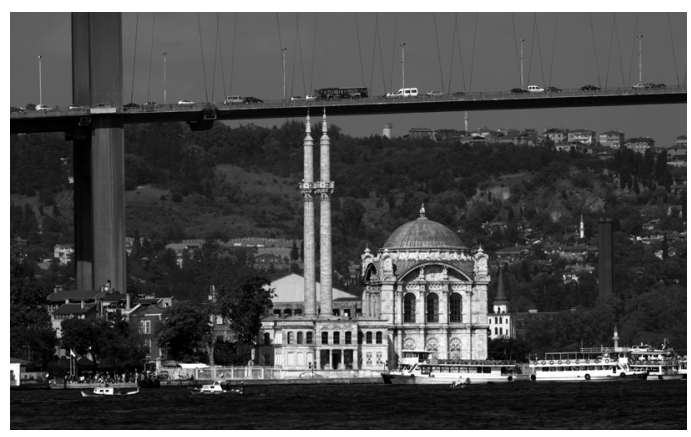


reich noch übrig geblieben war. Mit der Einbindung in eine völkerrechtliche Gemeinschaft, die sich ihrerseits stetig veränderte, geriet die neue Türkei in Abhängigkeit von einem Außen, ein Außen, das sie kaum erfassen und fixieren konnte, weil auch ein Selbst noch nicht entwickelt war.

Die staatlichen Grenzen wurden 1923 festgesetzt, ein Staatsvolk sollte seine Identität finden, eine Staatsmacht sich entwickeln und stabilisieren. Das zivile Leben wurde bis dahin geregelt durch die Normen und die Rechtsprechung einer islamischen Rechtsschule (Hanefitische Rechtsschule), das Neue, das begonnen hatte, zwang zu Reformen in diesem Bereich, auch das Recht musste neu werden.

Das ist der Hintergrund, vor dem die Übernahme des schweizerischen Zivilgesetzbuches stattgefunden hat.

\section{Die Entwicklungen bis 1974 im Eherecht}

Die »Übernahme « der familienrechtlichen Bestimmungen des schweizerischen ZGB führte dazu, dass Polygamie gesetzlich nicht mehr erlaubt war. Im Gegensatz zum Zustand vorher, namentlich der islamrechtlichen Zulässigkeit von Mehrehen, wurde nun die zivilrechtliche Einehe vorgeschrieben. Bereits bestehende Mehrehen wurden durch Übergangsbestimmungen von der Anwendung ausgenommen, doch alle zukünftigen Ehen hatten dieser zivilrechtlichen (oder sollte man lieber sagen: zivilgesetzlichen?) Vorgabe zu genügen. Das Verbot der Polygamie schien jedoch die Menschen nicht zu kümmern, im Gegenteil: Die alte Form der Eheschließung bestand fort. Weiterhin wurden Ehen vor einem islamischen Geistlichen, sei es einem `Hoca einem `Imam`, geschlossen. Und weiterhin konnte ein Mann mit mehreren Frauen islamrecht- liche eheliche Bindungen eingehen, ohne dass er Sanktionen zu befürchten hatte. Gesellschaftlich wurde die traditionelle Eheschließung anerkannt, gesetzlich zwar verboten, aber faktisch nicht bestraft.

Die Situation stellte sich alsbald folgendermaßen dar: Wenn gesetzlich nur die Einehe zulässig war und zivilrechtlich der Bund der Ehe vor einem Standesbeamten geschlossen und eingetragen werden musste, konnten all jene Fälle, die dieses Verfahren nicht durchliefen, bürokratisch nicht erfasst und rechtlich nicht reguliert werden. So lange aber die zivilrechtliche Zulässigkeit und Anerkennung der Ehe für die Betroffenen keine Relevanz hatte, stellte sich auch kein konkretes rechtliches Entscheidungsproblem: Die Geschichte spielte anderswo.

Dem Gesetzgeber blieb nur das Beobachten, »mitmachen « durfte er nicht. Prinzipiell stand er unter dem Druck zu handeln und in seinem Selbstverständnis als Gesetz-Geber in Erscheinung zu treten. Dabei traf er allerdings nicht einmal auf aktive Zurückweisung seitens der »Rechtsunterworfenen «. Vielmehr wurde er schlicht ignoriert und gar nicht wahrgenommen.

Die Gründe für die - andauernde - Beliebtheit der Mehrehe bedürfen im Einzelnen noch der sorgfältigen Untersuchung. Einen Hinweis auf die soziale und bevölkerungspolitische Bedeutung der Mehrehe gibt immerhin ein Zeitungsartikel im »Journal de Genève« vom I3. Februar I923, dessen Überschrift lautete "Le mariage obligatoire en Turquie? «. ${ }^{2}$ Der Verfasser handelte nicht etwa - im Vorgriff auf die zivilgesetzlichen Regeln von I926 - von der zukünftig einzig legalen und obligatorischen Eheschließung. Anliegen des Abgesandten von Erzurum, Khali Efendi, war vielmehr ein Gesetzesvorschlag, der die Dezimierung der Bevölkerung durch die Kriege ausgleichen sollte. Durch das Gesetz sollten

2 Journal de Genève vom I3. Februar 1923,3 . 
alle Unverheirateten spätestens mit dem 25. Lebensjahr eine Ehe eingehen; und damit nicht genug: "Tout célibataire de 25 ans devra se marier immédiatement et avoir au minimum un enfant tous les trois ans (sic!)." In diesem Licht erscheint die Einführung der Einehe nicht geeignet, um die türkische Bevölkerung zu vergröBern.

Kehren wir zurück zum Gesetzgeber, der noch keiner sein konnte: Dass die Ehen in einer anderen Form als der zivilrechtlichen geschlossen wurden, war ein Problem insofern, als sich diese Verbindungen eben dem Zivilrecht entzogen. Hinzu kam aber auch der Umstand, dass die Kinder, die aus solchen Verbindungen hervorgingen, nicht als Nachkommen im zivilrechtlichen Sinne erfasst werden konnten.

In regelmäßigen Abständen (1933, I945, I956, I965, ...) erließ der türkische Gesetzgeber nun also Sondergesetze, Gesetze mit zeitlich beschränkter Geltungsdauer, um dem beschriebenen Zustand im Eherecht und Kindesrecht abzuhelfen. I974, ein halbes Jahrhundert nach der Übernahme des ZGB, wurde ein solches Sondergesetz erlassen, das nähere Betrachtung verdient:

Mit dem Gesetz Nr. I826 wurde eine Art Wahlmöglichkeit geschaffen, islamrechtliche Ehen zu legalisieren. 'Außereheliche Verbindungen, also all die Beziehungen, die nicht das zivilrechtliche Verfahren durchlaufen hatten, konnten durch eine einfache Erklärung registriert werden, eine Art politische Legalisierung der Ehe wurde möglich. Kinderlose Paare allerdings, die in einer eheähnlichen Gemeinschaft lebten, konnten diese Sonderregelung nicht nutzen. Die Möglichkeit der Legalisierung stand also nur für Verbindungen offen, aus denen Kinder entstanden waren. Denn diese Kinder konnten ebenfalls durch eine einfache Erklärung an die Ver- waltungsbehörde als »ehelich « registriert werden. Dies erscheint auf den ersten Blick als nützlicher Akt des Gesetzgebers: Was außerhalb seiner Machtsphäre liegt, unterwirft sich selbst.

Mit dieser sondergesetzlichen Möglichkeit wurde jedoch gerade die Differenz erneuert, die überwunden werden sollte: »Für Türken besteht die Möglichkeit, sich in familienrechtlichen Fragen entweder dem sstatus traditionnek (status musulman) oder dem sstatus moderne (status civil) zu unterstellen. « ${ }^{3}$ Eine Ausschließlichkeit von Macht, ein Machtmonopol des Staates gab es nicht.

Zudem verschärfte sich die Problematik insofern, als die Polygamie dadurch nicht abgeschafft werden konnte, und die Schwierigkeit, Kinder als Kinder im zivilgesetzlichen Sinne zu qualifizieren, sich keineswegs auflöste, sondern sogar verstärkte. Lebte nun ein Mann polygam und hatte er Kinder aus den verschiedenen Verbindungen, so konnte durch das neue Gesetz eine dieser Verbindungen inklusive der Kinder registriert werden. Die anderen Beziehungen galten zivilrechtlich als Nichtehen. Aber: Der Mann, der eine Verbindung als Ehe registriert hatte, konnte alle seine Kinder, auch diejenigen außerhalb der registrierten Verbindung, als ehelich registrieren lassen, was zu einer Legitimation von Ehebruchskindern führte. Somit wurde ein Kind, das nicht aus der ehelichen Verbindung des Vaters stammte, trotzdem als sehelich erklärt und erlangte dadurch in der Erbfolge dieselbe Stellung wie die Kinder aus der registrierten Ehe. Dies im Verhältnis zum Vater in der legalisierten Ehe. Gleichzeitig waren diese Kinder aber auch Erben im Verhältnis zur leiblichen Mutter, obwohl diese nicht Teil der legitimierten Ehe war. Anders ausgedrückt: Kinder erhielten die (erbrechtliche) Stellung ehelicher Kinder gegenüber ihrem Vater und ihrer Mutter, obwohl ihre Eltern

3 Hilmar Krüger, Osmanischislamische Tradition versus Zivilgesetzbuch, in: Zeitschrift für schweizerisches Recht (ZSR) 95 I (I976) 287-30I, 289. 
weder nach Zivilrecht, noch nach Sonderrecht verheiratet waren.

Durch das Sondergesetz Nr. I826 wurde also ein Zustand herbeigeführt, der nicht neu war: Im islamisch-osmanischen Recht gilt das Kind immer als sehelich`, sobald ein Mann die Vaterschaft zu diesem Kind anerkennt (’nesebin ikrari< / iqrâr an-nasab), und in der Gesellschaft war eine solche Anerkennung nach wie vor möglich. Aufgrund dieses eigenartigen Zustands erschienen auch andere Regelungen in einem merkwürdigen Licht, etwa die elterliche Gewalt, das Sorgerecht und anderes mehr, worauf hier nicht im Einzelnen eingegangen werden kann.

\section{Die Revision des türkischen \\ Zivilgesetzbuches 200I}

Was 1926 begann - der Transfer des schweizerischen ZGB in die Türkei -, wurde, wie wir sahen, jedenfalls im Familien- und Eherecht mehrfach reformiert und reformuliert. Auch vor nun mehr als einem Vierteljahrhundert wurde mit dem Gesetz von 1976 ein weiterer Anlauf unternommen, das »importierte Recht den türkischen Verhältnissen anzupassen. Der Prozess des Transfers, so möchte man daraus schließen, war damals noch nicht abgeschlossen, womit sich die Frage stellt, wann ein solcher Prozess endet, wann ein Transfer vollzogen ist und eine eigene Gesetzgebung beginnt.

Im Falle der Türkei kann festgehalten werden, dass sich fast 80 Jahre nach »Übernahme « des schweizerischen Zivilgesetzbuches eine Eigenrechtlichkeit nicht eingestellt hat.

Am I. Januar 2002 ist das neue Zivilgesetzbuch in der Türkei in Kraft getreten. Eine Revision kann viele Motive haben. Sie kann gerade auch den Sprung in die Eigengesetzlichkeit markieren, kann einen Versuch darstellen, die Ge- setze den sozialen Veränderungen anzupassen oder - im Fall eines historischen Transfers nun das Fremde abzuschütteln. Ein Dreivierteljahrhundert, nachdem die Türkei ein fremdes Gesetzbuch übernommen hatte, liest man zur Revision:

»Das türkische Zivilgesetzbuch ist aufgrund von sozialen und ökonomischen Änderungen in der türkischen Gesellschaft [revidiert worden] und am I. Januar 2002 in Kraft getreten. Als Grundlage der Erneuerung diente das schweizerische Zivilgesetzbuch (ZGB)." Dieses Zitat stammt aus einem Aufsatz von Gezici/Kalkan ${ }^{4}$ aus dem Jahr 2002. Die türkische Gesellschaft ändert sich, die Anpassung der Gesetze erfolgt schweizerisch? Einmal mehr stellt sich die Frage: Weshalb wieder eine Referenz »Schweiz «? Und was bedeutet eine solche Rückfahrkarte, wenn sie 74 Jahre nach dem ersten Transfer eingelöst wird? Um dieses Rätsel zu lösen, mögen die folgenden Hypothesen einen ersten Ansatzpunkt bieten.

- Die familienrechtlichen Normen des ZGB haben im Fall der Türkei keine Irritation des Rechts bewirkt.

- Die Entwicklungen sind ähnlich denen, die Eugen Ehrlich ${ }^{5}$ beschrieben hat. Mag das schweizerische Zivilgesetzbuch übernommen worden und politisch in Kraft gesetzt worden sein, um türkisches Recht zu werden. Im Bereich des Familienrechts hat es diesen Weg nicht geschafft: Es blieb an der Peripherie des Rechts, es blieb formal und es blieb »totes Recht $«$.

- Die Kommunikationszusammenhänge des Rechts, das »lebendige " Recht, entwickelten sich » ungestört « weiter.

- Ob sich die weitere Entwicklung anders gestalten wird, ob also Irritationen auftreten, muss sich noch zeigen. Die Revision des ZGB,

4 Sevim Gezici u. Burcu Kalkan, Änderungen innerhalb des Eheschließungsrechts im türkischen Zivilrecht, in: Annales de la Faculté de Droit d'Istanbul 5I (2002) 277-298, 277.

5 Eugen Ehrlich, Gesetz und lebendes Recht. Vermischte kleine Schriften hg. von Manfred ReHBINDER, Berlin I 986. 
die auch die familienrechtlichen Normen betrifft, lässt das Experiment von neuem beginnen.

- An einem Makel scheint der neue Versuch jedoch bereits jetzt zu leiden: Er schließt just an das an, was nicht zu stören vermochte. Der Grund dafür dürfte in Legitimation durch Tradition liegen.

Vergleicht man diese Hypothesen mit den Positionen von Pierre Legrand einerseits, der "legal transplants « für unmöglich hält, und Alan Watson andererseits, der solche bejaht, ergibt sich Folgendes: Das Scheitern des Transfers Schweiz-Türkei stützt prima facie die Ansicht von Pierre Legrand. Doch ist zu bedenken, dass das Familienrecht einen ganz besonderen Fall für einen Rechtstransfer darstellt. Der Transfer soll in einem kulturell hoch determinierten Bereich stattfinden, in einem Bereich, in dem Recht mit dem sozialen Leben, seinen Gewohnheiten, Herkommen, religiösen Gebräuchen aufs Engste verbunden ist. Zudem handelt es sich im Fall Schweiz-Türkei um einen Transfer von einer funktional ausdifferenzierten Gesellschaft in eine vermutungsweise stratifizierte Gesellschaft. $\mathrm{Ob}$ Transfers zwischen unterschiedlich strukturierten Gesellschaften überhaupt möglich sind, bliebe zu untersuchen.

Selbst wenn sich mein Verdacht, dass der Transfer im Bereich des Familienrechts missglückt ist, erhärten sollte, scheint doch eines augenfällig: Durch den Transferversuch, durch die politische Übernahme von Gesetzen, ist jedenfalls ein Prozess in Gang gesetzt worden, etwa im Bereich des Familienrechts die aufgezeig- ten gesetzgeberischen Aktivitäten. Etwas bewegt sich doch - auch im Fall des fehlgeschlagenen Transfers. Und so treibt - im Sinne Stichwehs ${ }^{6}$ der transferierte Replikator auch ohne Replikation die Evolution voran, wohin auch immer.

Auch Eugen Ehrlich wies auf diesen Wert eines Rechtstransfers hin. Mag bei ihm die Diskussion darum kreisen, ob das Recht, das übernommen wird, wirklich national sei, und möge er zum Schluss kommen, dass Gesetzbücher »... unzählige Male durchsiebte Abstraktionen und Verallgemeinerungen [enthalten] «, ${ }^{7}$ dass die Verbundenheit mit der Quelle des nationalen Lebens nicht mehr gegeben ist und somit von Anfang an etwas transferiert wird, das gar nicht das ist, was es zu sein scheint, nämlich im Beispiel Ehrlichs das undeutsche sdeutsche BGB nach Japan, angewandt auf unseren Fall das sunschweizerische schweizerische ZGB in die Türkei. Dennoch solle damit "... keineswegs behauptet werden, dass das Gesetzbuch wertlos sei «, ${ }^{8}$ die Übernahme eines Gesetzbuches könne große Dienste für die entsprechende Rechtswissenschaft, die Rechtspflege, auch den Rechtsunterricht leisten, da eine Auseinandersetzung mit dem Transferierten erfolgt; es könne auch $\mathrm{zu}$ einer brauchbaren Grundlage für künftige Gesetzgebung werden. Mit dem nicht genug: »[Es] ... erwächst die ungemein wichtige Aufgabe, sich mit der Kenntnisnahme des Gesetzbuches nicht zu begnügen, sondern die gesellschaftliche Ordnung $\mathrm{zu}$ erforschen, die den [eigenen] nationalen Hintergrund [des übernommenen] Gesetzbuches bildet. «9

Mahidé Aslan

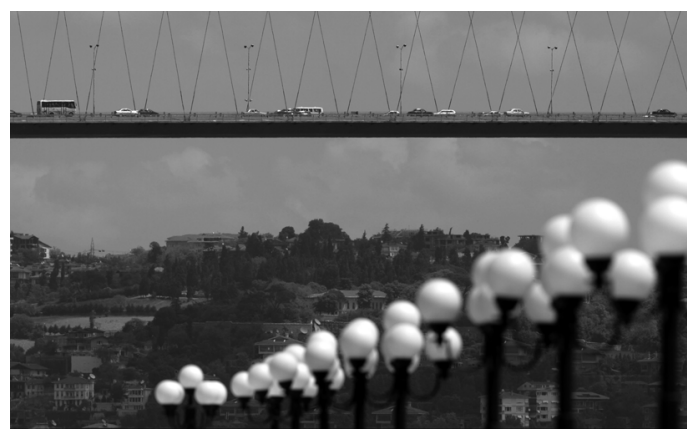

6 Sinngemäß im Vortrag "Transfer in Sozialsystemen « von Rudolf Stichweh, gehalten anlässlich der Eröffnung des Europäischen Forums junger Rechtshistorikerinnen und Rechtshistoriker vom 26. -29. Mai 2005 in Luzern.

7 Ehrlich (Fn. 5) 236.

8 Ehrlich (Fn. 5) 236.

9 Ehrlich (Fn. 5) 236. 